STRUCTURE REPORTS

ISSN 1600-5368

\section{5-Chloro-3-(4-fluorophenylsulfonyl)-2,7- dimethyl-1-benzofuran}

\author{
Hong Dae Choi ${ }^{\mathrm{a}}$ and Uk Lee $\mathrm{b}^{\mathrm{b} *}$ \\ a Department of Chemistry, Dongeui University, San 24 Kaya-dong, Busanjin-gu, \\ Busan 614-714, Republic of Korea, and ${ }^{\mathbf{b}}$ Department of Chemistry, Pukyong \\ National University, 599-1 Daeyeon 3-dong, Nam-gu, Busan 608-737, Republic of \\ Korea. *Correspondence e-mail: uklee@pknu.ac.kr
}

Received 5 August 2014; accepted 24 August 2014

Edited by J. T. Mague, Tulane University, USA

In the title compound, $\mathrm{C}_{16} \mathrm{H}_{12} \mathrm{ClFO}_{3} \mathrm{~S}$, the dihedral angle between the plane of the benzofuran ring system [r.m.s. deviation $=0.007$ (1) $\AA$ ] and that of the 4-fluorophenyl ring is $76.11(5)^{\circ}$. In the crystal, molecules are linked into [010] chains via two different inversion-generated pairs of $\mathrm{C}-\mathrm{H} \cdots \mathrm{O}$ hydrogen bonds. The crystal structure also exhibits weak $\pi-\pi$ interactions between the benzene and furan rings of neighbouring molecules [centroid-centroid distance = 3.820 (2) A] .

Keywords: crystal structure; benzofuran; 4-fluorophenyl; $\mathrm{C}-\mathrm{H} \ldots \mathrm{O}$ hydrogen bonds; $\pi-\pi$ interactions.

CCDC reference: 1020842

\section{Related literature}

For the pharmaceutical properties of compounds containing benzofuran moieties, see: Aslam et al. (2009); Galal et al. (2009); Howlett et al. (1999); Khan et al. (2005); Ono et al. (2002). For natural products with a benzofuran ring, see: Akgul \& Anil (2003); Soekamto et al. (2003). For the synthesis of the starting material 5-chloro-3-(4-fluorophenylsulfanyl)2,7-dimethyl-1-benzofuran, see: Choi et al. (1999). For a related structure, see: Choi et al. (2014).

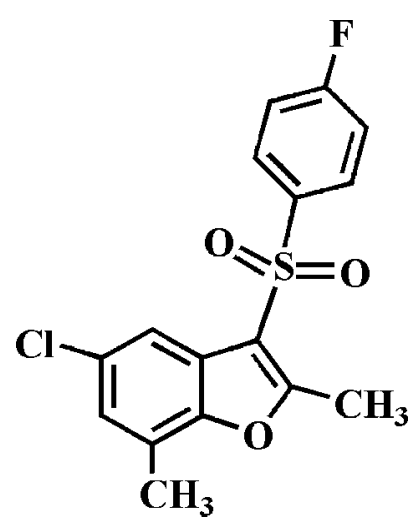

\section{Experimental}

\subsection{Crystal data}

$$
\begin{aligned}
& \mathrm{C}_{16} \mathrm{H}_{12} \mathrm{ClFO}_{3} \mathrm{~S} \\
& M_{r}=338.77 \\
& \text { Triclinic, } P \overline{1} \\
& a=8.4338(3) \AA \\
& b=9.9171(3) \AA \\
& c=10.1059(3) \AA \\
& \alpha=73.988(2)^{\circ} \\
& \beta=66.155(2)^{\circ}
\end{aligned}
$$

$$
\begin{aligned}
& \gamma=73.629(2)^{\circ} \\
& V=729.02(4) \AA^{3} \\
& Z=2 \\
& \text { Mo } K \alpha \text { radiation } \\
& \mu=0.43 \mathrm{~mm}^{-1} \\
& T=173 \mathrm{~K} \\
& 0.47 \times 0.31 \times 0.15 \mathrm{~mm}
\end{aligned}
$$

\subsection{Data collection}

Bruker SMART APEXII CCD diffractometer

Absorption correction: multi-scan (SADABS; Bruker, 2009)

$T_{\min }=0.825, T_{\max }=0.939$

\subsection{Refinement}

$R\left[F^{2}>2 \sigma\left(F^{2}\right)\right]=0.037$

$w R\left(F^{2}\right)=0.098$

$S=1.03$

3614 reflections

13460 measured reflections 3614 independent reflections 3163 reflections with $I>2 \sigma(I)$ $R_{\text {int }}=0.027$

Table 1 Hydrogen-bond geometry $\left(\AA{ }^{\circ}\right)$.

\begin{tabular}{lllll}
\hline$D-\mathrm{H} \cdots A$ & $D-\mathrm{H}$ & $\mathrm{H} \cdots A$ & $D \cdots A$ & $D-\mathrm{H} \cdots A$ \\
\hline $\mathrm{C} 9-\mathrm{H} 9 C \cdots \mathrm{O} 2^{\mathrm{i}}$ & 0.98 & 2.57 & $3.331(2)$ & 135 \\
$\mathrm{C} 16-\mathrm{H} 16 \cdots \mathrm{O} 3^{\mathrm{ii}}$ & 0.95 & 2.53 & $3.230(2)$ & 130 \\
\hline
\end{tabular}

Symmetry codes: (i) $-x,-y+1,-z+1$; (ii) $-x,-y+2,-z$.

Data collection: APEX2 (Bruker, 2009); cell refinement: SAINT (Bruker, 2009); data reduction: $S A I N T$; program(s) used to solve structure: SHELXS97 (Sheldrick, 2008); program(s) used to refine structure: SHELXL97 (Sheldrick, 2008); molecular graphics: ORTEP-3 for Windows (Farrugia, 2012) and DIAMOND (Brandenburg, 1998); software used to prepare material for publication: SHELXL97.

\section{Acknowledgements}

The X-ray centre of the Gyeongsang National University is acknowledged for providing access to the single-crystal diffractometer. 
Supporting information for this paper is available from the IUCr electronic archives (Reference: MW2126).

\section{References}

Akgul, Y. Y. \& Anil, H. (2003). Phytochemistry, 63, 939-943.

Aslam, S. N., Stevenson, P. C., Kokubun, T. \& Hall, D. R. (2009). Microbiol. Res. 164, 191-195.

Brandenburg, K. (1998). DIAMOND. Crystal Impact GbR, Bonn, Germany. Bruker (2009). APEX2, SADABS and SAINT. Bruker AXS Inc., Madison, Wisconsin, USA.

Choi, H. D., Seo, P. J. \& Lee, U. (2014). Acta Cryst. E70, o568.
Choi, H. D., Seo, P. J. \& Son, B. W. (1999). J. Korean Chem. Soc. 43, 606608.

Farrugia, L. J. (2012). J. Appl. Cryst. 45, 849-854.

Galal, S. A., Abd El-All, A. S., Abdallah, M. M. \& El-Diwani, H. I. (2009). Bioorg. Med. Chem. Lett. 19, 2420-2428.

Howlett, D. R., Perry, A. E., Godfrey, F., Swatton, J. E., Jennings, K. H., Spitzfaden, C., Wadsworth, H., Wood, S. J. \& Markwell, R. E. (1999). Biochem. J. 340, 283-289.

Khan, M. W., Alam, M. J., Rashid, M. A. \& Chowdhury, R. (2005). Bioorg. Med. Chem. 13, 4796-4805.

Ono, M., Kung, M. P., Hou, C. \& Kung, H. F. (2002). Nucl. Med. Biol. 29, 633642.

Sheldrick, G. M. (2008). Acta Cryst. A64, 112-122.

Soekamto, N. H., Achmad, S. A., Ghisalberti, E. L., Hakim, E. H. \& Syah, Y. M. (2003). Phytochemistry, 64, 831-834. 


\section{supporting information}

Acta Cryst. (2014). E70, o1065-o1066 [doi:10.1107/S1600536814019114]

\section{5-Chloro-3-(4-fluorophenylsulfonyl)-2,7-dimethyl-1-benzofuran}

\section{Hong Dae Choi and Uk Lee}

\section{S1. Comment}

Molecules containing the benzofuran skeleton show interesting pharmacological properties such as antibacterial, antifungal, antitumor, antiviral and antimicrobial activities (Aslam et al. 2009, Galal et al., 2009, Khan et al., 2005) as well as being potential inhibitors of $\beta$-amyloid aggregation (Howlett et al., 1999, Ono et al., 2002). These benzofuran compounds are widely occurring in nature (Akgul \& Anil, 2003, Soekamto et al., 2003). As a part of our ongoing project of 3-arylsulfonyl-5-chloro-2,7-dimethyl-1-benzofuran derivatives containing a 3-methylphenylsulfonyl substituent in the 3-position (Choi et al., 2014), we report herein on the crystal structure of the title compound.

In the title molecule (Fig. 1), the benzofuran unit is essentially planar, with a mean deviation of 0.007 (1) $\AA$ from the least-squares plane defined by the nine constituent atoms. The 4-fluorophenyl ring is essentially planar, with a mean deviation of 0.003 (1) $\AA$ from the least-squares plane defined by the six constituent atoms. The dihedral angle formed by the benzofuran ring system and the 4-fluorophenyl ring is $76.11(5)^{\circ}$. In the crystal structure (Fig. 2), molecules are linked via pairs of $\mathrm{C}-\mathrm{H} \cdots \mathrm{O}$ hydrogen bonds (Table 1), forming inversion dimers. The crystal packing (Fig. 2) also exhibits weak $\pi-\pi$ interactions between the benzene and furan rings of neighbouring molecules, with a $\mathrm{Cg} 1 \cdots \mathrm{Cg} 2^{\mathrm{i}}$ (i: $\left.-x, 1-y, 1-z\right)$ distance of 3.820 (2) $\AA$ and an interplanar distance of 3.641 (2) $\AA$ resulting in a slippage of 1.159 (2) $\AA$ ( $\mathrm{Cg} 1$ and $\mathrm{Cg} 2$ are the centroids of the $\mathrm{C} 2-\mathrm{C} 7$ benzene ring and the $\mathrm{C} 1 / \mathrm{C} 2 / \mathrm{C} 7 / \mathrm{O} 1 / \mathrm{C} 8$ furan ring, respectively),

\section{S2. Experimental}

The starting material 5-chloro-3-(4-fluorophenylsulfanyl)-2,7-dimethyl-1-benzofuran was prepared by the literature method (Choi et al., 1999). 3-Chloroperoxybenzoic acid (77\%, $515 \mathrm{mg}, 2.3 \mathrm{mmol})$ was added in small portions to a stirred solution of 5-chloro-3-(4-fluorophenylsulfanyl)-2,7-dimethyl-1-benzofuran (337 mg, $1.1 \mathrm{mmol}$ ) in dichloromethane $(35 \mathrm{~mL})$ at $273 \mathrm{~K}$. After being stirred at room temperature for $10 \mathrm{~h}$, the mixture was washed with saturated sodium bicarbonate solution $(2 \times 20 \mathrm{~mL})$ and the organic layer was separated, dried over magnesium sulfate, filtered and concentrated at reduced pressure. The residue was purified by column chromatography (hexane-ethyl acetate, $4: 1 \mathrm{v} / \mathrm{v}$ ) to afford the title compound as a colorless solid [yield 71\% (240 mg); m.p. 468-469 K; $R_{\mathrm{f}}=0.56$ (hexane-ethyl acetate, 4:1 $v / v)$ ]. Single crystals suitable for X-ray diffraction were prepared by slow evaporation of a solution of the title compound $(30 \mathrm{mg})$ in ethyl acetate $(20 \mathrm{~mL})$ at room temperature.

\section{S3. Refinement}

All $\mathrm{H}$ atoms were positioned geometrically and refined using a riding model, with $\mathrm{C}-\mathrm{H}=0.95 \AA$ for aryl and $0.98 \AA$ for methyl $\mathrm{H}$ atoms, $U_{\text {iso }}(\mathrm{H})=1.2 U_{\text {eq }}(\mathrm{C})$ for aryl and $1.5 U_{\text {eq }}(\mathrm{C})$ for methyl $\mathrm{H}$ atoms. The positions of methyl hydrogens were optimized using the SHELXL-97 command AFIX 137 (Sheldrick, 2008). 


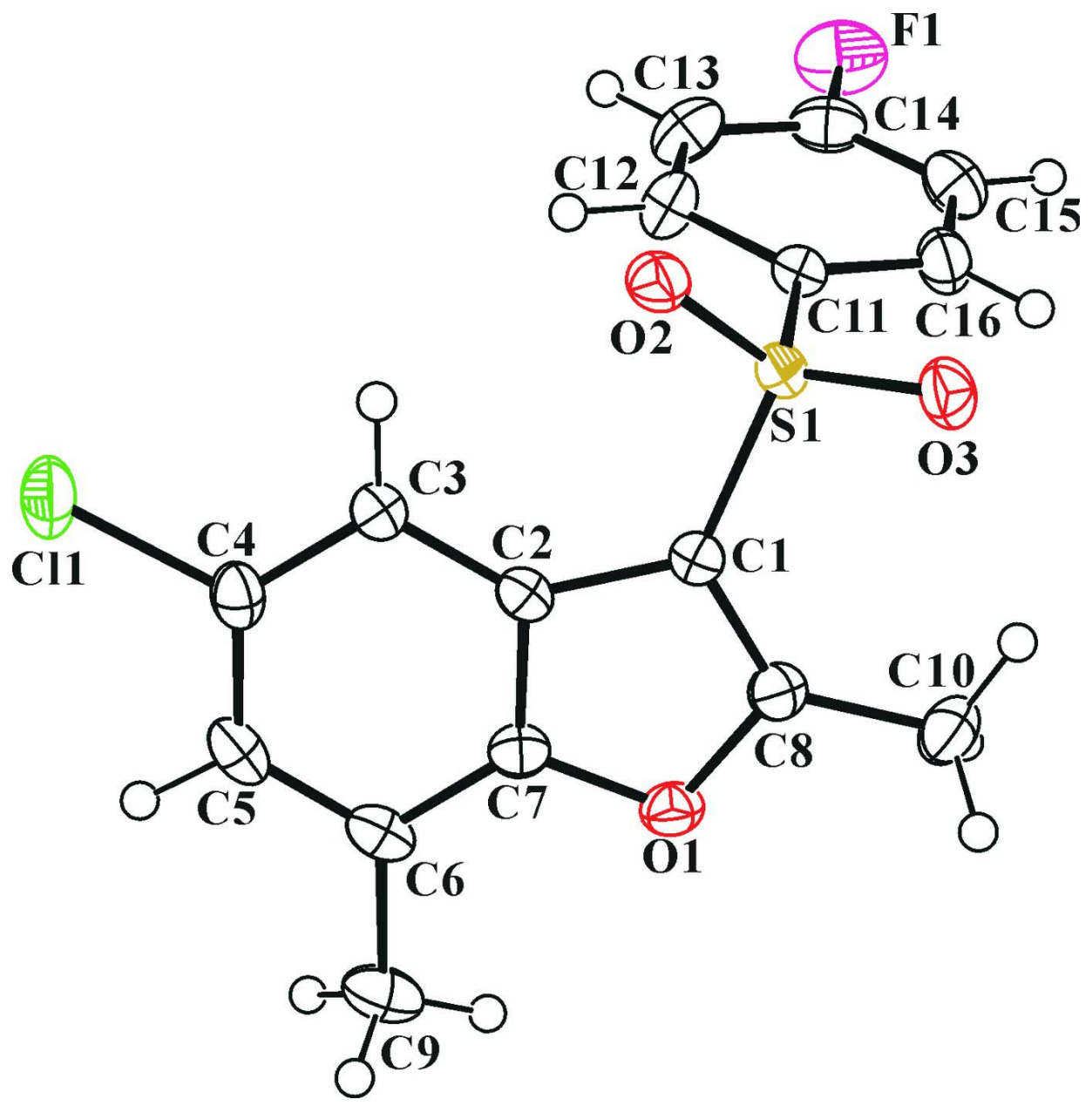

\section{Figure 1}

The molecular structure of the title molecule with the atom numbering scheme The displacement ellipsoids are drawn at the $50 \%$ probability level. The hydrogen atoms are presented as small spheres of arbitrary radius. 


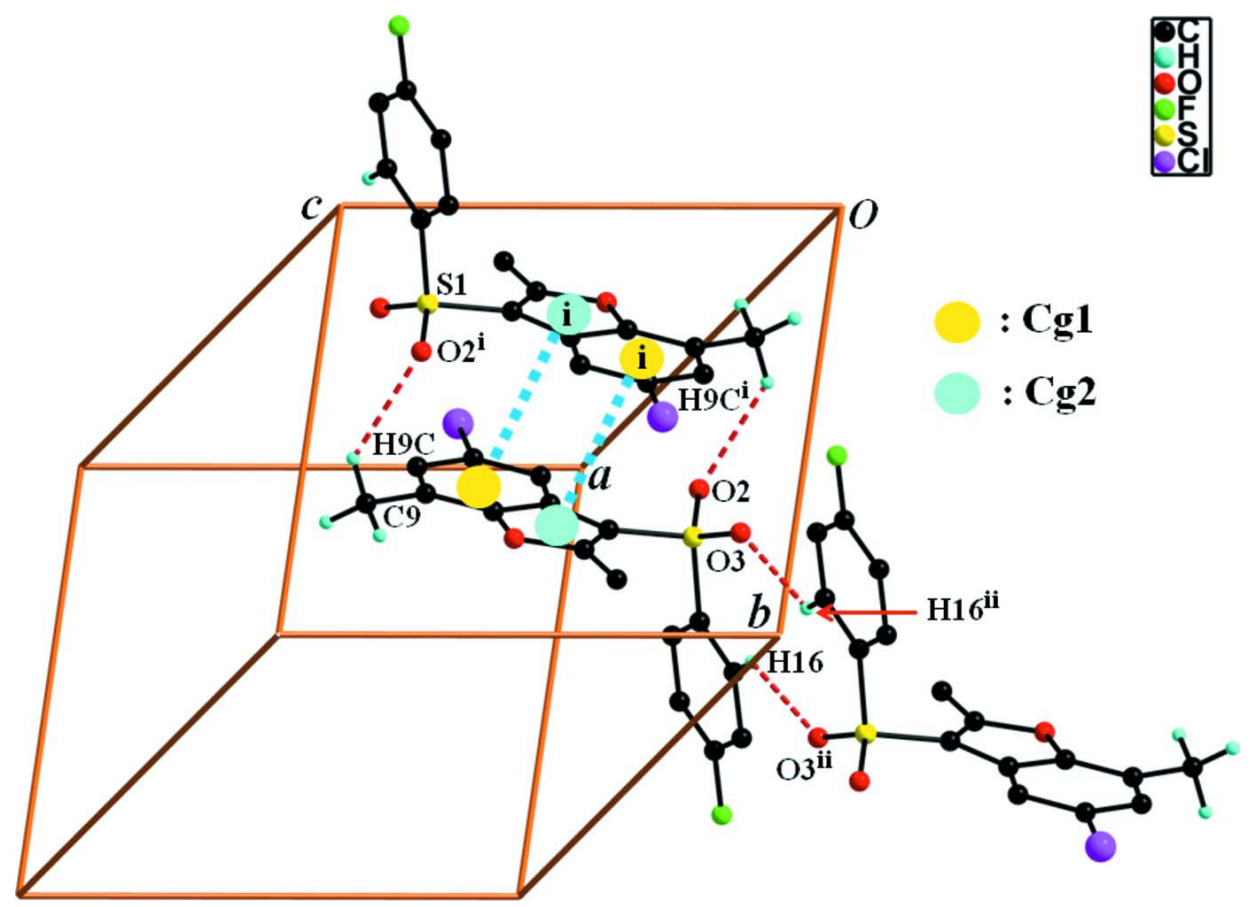

\section{Figure 2}

A view of the $\mathrm{C}-\mathrm{H} \cdots \mathrm{O}$ and $\pi-\pi$ interactions (dotted lines) in the crystal structure of the title compound. $\mathrm{H}$ atoms nonparticipating in hydrogen-bonding were omitted for clarity. [Symmetry codes: (i) $-x,-y+1,-z+1$; (ii) $-x,-y+2,-z$.]

\section{5-Chloro-3-(4-fluorophenylsulfonyl)-2,7-dimethyl-1-benzofuran}

\section{Crystal data}

\section{$\mathrm{C}_{16} \mathrm{H}_{12} \mathrm{ClFO}_{3} \mathrm{~S}$}

$M_{r}=338.77$

Triclinic, $P \overline{1}$

Hall symbol: -P 1

$a=8.4338(3) \AA$

$b=9.9171(3) \AA$

$c=10.1059$ (3) $\AA$

$\alpha=73.988(2)^{\circ}$

$\beta=66.155(2)^{\circ}$

$\gamma=73.629(2)^{\circ}$

$V=729.02(4) \AA^{3}$

\section{Data collection}

\section{Bruker SMART APEXII CCD}

diffractometer

Radiation source: rotating anode

Graphite multilayer monochromator

Detector resolution: 10.0 pixels $\mathrm{mm}^{-1}$

$\varphi$ and $\omega$ scans

Absorption correction: multi-scan

(SADABS; Bruker, 2009)

$T_{\min }=0.825, T_{\max }=0.939$

\section{$Z=2$}

$F(000)=348$

$D_{\mathrm{x}}=1.543 \mathrm{Mg} \mathrm{m}^{-3}$

Melting point $=469-468 \mathrm{~K}$

Mo $K \alpha$ radiation, $\lambda=0.71073 \AA$

Cell parameters from 5949 reflections

$\theta=2.2-28.3^{\circ}$

$\mu=0.43 \mathrm{~mm}^{-1}$

$T=173 \mathrm{~K}$

Block, colourless

$0.47 \times 0.31 \times 0.15 \mathrm{~mm}$

13460 measured reflections

3614 independent reflections

3163 reflections with $I>2 \sigma(I)$

$R_{\text {int }}=0.027$

$\theta_{\max }=28.3^{\circ}, \theta_{\min }=2.2^{\circ}$

$h=-10 \rightarrow 11$

$k=-13 \rightarrow 13$

$l=-13 \rightarrow 13$ 


\section{Refinement}

Refinement on $F^{2}$

Least-squares matrix: full

$R\left[F^{2}>2 \sigma\left(F^{2}\right)\right]=0.037$

$w R\left(F^{2}\right)=0.098$

$S=1.03$

3614 reflections

202 parameters

0 restraints

Primary atom site location: structure-invariant direct methods
Secondary atom site location: difference Fourier map

Hydrogen site location: difference Fourier map

$\mathrm{H}$-atom parameters constrained

$w=1 /\left[\sigma^{2}\left(F_{\mathrm{o}}^{2}\right)+(0.0444 P)^{2}+0.4675 P\right]$

where $P=\left(F_{\mathrm{o}}^{2}+2 F_{\mathrm{c}}^{2}\right) / 3$

$(\Delta / \sigma)_{\max }=0.001$

$\Delta \rho_{\max }=0.34$ e $\AA^{-3}$

$\Delta \rho_{\min }=-0.37$ e $\AA^{-3}$

Extinction correction: SHELXL97 (Sheldrick, 2008), $\mathrm{Fc}^{*}=\mathrm{kFc}\left[1+0.001 \times \mathrm{xc}^{2} \lambda^{3} / \sin (2 \theta)\right]^{-1 / 4}$

Extinction coefficient: 0.016 (2)

\section{Special details}

Experimental. 1H NMR ( $\delta$ p.p.m., $\left.\mathrm{CDCl}_{3}, 400 \mathrm{~Hz}\right)$ : 7.98-8.03 (m, 2H), $7.67(\mathrm{~d}, \mathrm{~J}=2.04 \mathrm{~Hz}, 1 \mathrm{H}), 7.17-7.22(\mathrm{~m}, 2 \mathrm{H})$, $7.10(\mathrm{~s}, 1 \mathrm{H}), 2.80(\mathrm{~s}, 3 \mathrm{H}), 2.43(\mathrm{~s}, 3 \mathrm{H})$.

Geometry. All esds (except the esd in the dihedral angle between two 1.s. planes) are estimated using the full covariance matrix. The cell esds are taken into account individually in the estimation of esds in distances, angles and torsion angles; correlations between esds in cell parameters are only used when they are defined by crystal symmetry. An approximate (isotropic) treatment of cell esds is used for estimating esds involving l.s. planes.

Refinement. Refinement of $\mathrm{F}^{2}$ against ALL reflections. The weighted R-factor $\mathrm{wR}$ and goodness of fit $\mathrm{S}$ are based on $\mathrm{F}^{2}$, conventional R-factors $R$ are based on $F$, with $F$ set to zero for negative $F^{2}$. The threshold expression of $F^{2}>2 \operatorname{sigma}\left(\mathrm{F}^{2}\right)$ is used only for calculating R-factors(gt) etc. and is not relevant to the choice of reflections for refinement. R-factors based on $\mathrm{F}^{2}$ are statistically about twice as large as those based on F, and R- factors based on ALL data will be even larger.

Fractional atomic coordinates and isotropic or equivalent isotropic displacement parameters $\left(\hat{A}^{2}\right)$

\begin{tabular}{lllll}
\hline & $x$ & $y$ & $z$ & $U_{\text {iso }} * / U_{\text {eq }}$ \\
\hline C11 & $0.51698(6)$ & $0.19310(5)$ & $0.47396(5)$ & $0.03701(14)$ \\
S1 & $0.13342(5)$ & $0.69006(4)$ & $0.14183(4)$ & $0.02143(12)$ \\
F1 & $0.70966(17)$ & $0.98587(15)$ & $-0.25209(14)$ & $0.0518(4)$ \\
O1 & $0.04838(15)$ & $0.74576(13)$ & $0.53640(13)$ & $0.0263(3)$ \\
O2 & $0.18910(16)$ & $0.54497(12)$ & $0.11860(13)$ & $0.0272(3)$ \\
O3 & $-0.02442(16)$ & $0.77606(13)$ & $0.11823(13)$ & $0.0291(3)$ \\
C1 & $0.1165(2)$ & $0.68330(17)$ & $0.32016(17)$ & $0.0215(3)$ \\
C2 & $0.2069(2)$ & $0.56854(17)$ & $0.40420(17)$ & $0.0217(3)$ \\
C3 & $0.3177(2)$ & $0.43625(17)$ & $0.38227(18)$ & $0.0235(3)$ \\
H3 & 0.3506 & 0.4013 & 0.2943 & $0.028 *$ \\
C4 & $0.3767(2)$ & $0.35884(18)$ & $0.49555(19)$ & $0.0260(3)$ \\
C5 & $0.3304(2)$ & $0.40691(19)$ & $0.62598(19)$ & $0.0285(4)$ \\
H5 & 0.3761 & 0.3492 & 0.6996 & $0.034 *$ \\
C6 & $0.2188(2)$ & $0.53740(19)$ & $0.65043(18)$ & $0.0267(4)$ \\
C7 & $0.1604(2)$ & $0.61368(18)$ & $0.53606(18)$ & $0.0236(3)$ \\
C8 & $0.0241(2)$ & $0.78571(18)$ & $0.40404(18)$ & $0.0240(3)$ \\
C9 & $0.1674(3)$ & $0.5919(2)$ & $0.78961(19)$ & $0.0361(4)$ \\
H9A & 0.1210 & 0.6950 & 0.7737 & $0.054 *$ \\
H9B & 0.2713 & 0.5730 & 0.8175 & $0.054^{*}$ \\
H9C & 0.0764 & 0.5431 & 0.8685 & $0.054^{*}$ \\
C10 & $-0.0915(2)$ & $0.92612(19)$ & $0.3815(2)$ & $0.0322(4)$ \\
& & & &
\end{tabular}




$\begin{array}{lllll}\text { H10A } & -0.0361 & 1.0018 & 0.3790 & 0.048^{*} \\ \text { H10B } & -0.2059 & 0.9286 & 0.4625 & 0.048^{*} \\ \text { H10C } & -0.1092 & 0.9411 & 0.2881 & 0.048^{*} \\ \text { C11 } & 0.3084(2) & 0.78021(17) & 0.02639(17) & 0.0224(3) \\ \text { C12 } & 0.4815(2) & 0.70757(19) & 0.0003(2) & 0.0306(4) \\ \text { H12 } & 0.5062 & 0.6108 & 0.0468 & 0.037^{*} \\ \text { C13 } & 0.6181(3) & 0.7776(2) & -0.0943(2) & 0.0374(4) \\ \text { H13 } & 0.7379 & 0.7301 & -0.1138 & 0.045^{*} \\ \text { C14 } & 0.5764(3) & 0.9174(2) & -0.1594(2) & 0.0339(4) \\ \text { C15 } & 0.4069(3) & 0.9914(2) & -0.1352(2) & 0.0348(4) \\ \text { H15 } & 0.3834 & 1.0880 & -0.1825 & 0.042^{*} \\ \text { C16 } & 0.2703(2) & 0.92165(19) & -0.03976(19) & 0.0299(4) \\ \text { H16 } & 0.1511 & 0.9706 & -0.0198 & 0.036^{*}\end{array}$

Atomic displacement parameters $\left(\AA^{2}\right)$

\begin{tabular}{lllllll}
\hline & $U^{11}$ & $U^{22}$ & $U^{33}$ & $U^{12}$ & $U^{13}$ & $U^{23}$ \\
\hline C11 & $0.0373(3)$ & $0.0245(2)$ & $0.0429(3)$ & $-0.00018(18)$ & $-0.0175(2)$ & $0.00258(18)$ \\
S1 & $0.0238(2)$ & $0.0211(2)$ & $0.01966(19)$ & $-0.00243(15)$ & $-0.00994(15)$ & $-0.00263(14)$ \\
F1 & $0.0438(7)$ & $0.0607(9)$ & $0.0443(7)$ & $-0.0292(7)$ & $0.0018(5)$ & $-0.0068(6)$ \\
O1 & $0.0261(6)$ & $0.0302(6)$ & $0.0231(6)$ & $-0.0035(5)$ & $-0.0074(5)$ & $-0.0100(5)$ \\
O2 & $0.0366(7)$ & $0.0233(6)$ & $0.0244(6)$ & $-0.0044(5)$ & $-0.0133(5)$ & $-0.0062(5)$ \\
O3 & $0.0253(6)$ & $0.0319(7)$ & $0.0299(6)$ & $-0.0029(5)$ & $-0.0148(5)$ & $-0.0005(5)$ \\
C1 & $0.0220(7)$ & $0.0222(8)$ & $0.0199(7)$ & $-0.0032(6)$ & $-0.0077(6)$ & $-0.0042(6)$ \\
C2 & $0.0229(7)$ & $0.0233(8)$ & $0.0186(7)$ & $-0.0066(6)$ & $-0.0072(6)$ & $-0.0015(6)$ \\
C3 & $0.0250(8)$ & $0.0225(8)$ & $0.0216(8)$ & $-0.0042(6)$ & $-0.0082(6)$ & $-0.0025(6)$ \\
C4 & $0.0252(8)$ & $0.0218(8)$ & $0.0285(8)$ & $-0.0057(6)$ & $-0.0106(6)$ & $0.0022(6)$ \\
C5 & $0.0307(9)$ & $0.0319(9)$ & $0.0234(8)$ & $-0.0122(7)$ & $-0.0135(7)$ & $0.0063(7)$ \\
C6 & $0.0280(8)$ & $0.0350(9)$ & $0.0190(7)$ & $-0.0138(7)$ & $-0.0076(6)$ & $-0.0010(6)$ \\
C7 & $0.0221(8)$ & $0.0264(8)$ & $0.0217(8)$ & $-0.0063(6)$ & $-0.0053(6)$ & $-0.0056(6)$ \\
C8 & $0.0224(8)$ & $0.0262(8)$ & $0.0245(8)$ & $-0.0045(6)$ & $-0.0086(6)$ & $-0.0062(6)$ \\
C9 & $0.0388(10)$ & $0.0529(12)$ & $0.0211(8)$ & $-0.0162(9)$ & $-0.0100(7)$ & $-0.0075(8)$ \\
C10 & $0.0290(9)$ & $0.0281(9)$ & $0.0395(10)$ & $0.0031(7)$ & $-0.0131(8)$ & $-0.0134(8)$ \\
C11 & $0.0243(8)$ & $0.0240(8)$ & $0.0188(7)$ & $-0.0036(6)$ & $-0.0079(6)$ & $-0.0042(6)$ \\
C12 & $0.0262(9)$ & $0.0245(9)$ & $0.0385(10)$ & $0.0002(7)$ & $-0.0105(7)$ & $-0.0084(7)$ \\
C13 & $0.0257(9)$ & $0.0379(11)$ & $0.0455(11)$ & $-0.0041(8)$ & $-0.0049(8)$ & $-0.0168(9)$ \\
C14 & $0.0347(10)$ & $0.0410(11)$ & $0.0260(9)$ & $-0.0166(8)$ & $-0.0031(7)$ & $-0.0084(8)$ \\
C15 & $0.0421(11)$ & $0.0321(10)$ & $0.0291(9)$ & $-0.0124(8)$ & $-0.0145(8)$ & $0.0045(7)$ \\
C16 & $0.0299(9)$ & $0.0277(9)$ & $0.0290(9)$ & $-0.0034(7)$ & $-0.0131(7)$ & $0.0012(7)$ \\
& & & & & & \\
\hline & & & & & &
\end{tabular}

Geometric parameters $\left(\stackrel{A}{ }{ }^{\circ}\right)$

\begin{tabular}{llll}
\hline $\mathrm{C} 11-\mathrm{C} 4$ & $1.7440(18)$ & $\mathrm{C} 6-\mathrm{C} 9$ & $1.501(2)$ \\
$\mathrm{S} 1-\mathrm{O} 3$ & $1.4356(12)$ & $\mathrm{C} 8-\mathrm{C} 10$ & $1.477(2)$ \\
$\mathrm{S} 1-\mathrm{O} 2$ & $1.4361(12)$ & $\mathrm{C} 9-\mathrm{H} 9 \mathrm{~A}$ & 0.9800 \\
$\mathrm{~S} 1-\mathrm{C} 1$ & $1.7332(16)$ & $\mathrm{C} 9-\mathrm{H} 9 \mathrm{~B}$ & 0.9800 \\
$\mathrm{~S} 1-\mathrm{C} 11$ & $1.7648(16)$ & $\mathrm{C} 9-\mathrm{H} 9 \mathrm{C}$ & 0.9800 \\
$\mathrm{~F} 1-\mathrm{C} 14$ & $1.353(2)$ & $\mathrm{C} 10-\mathrm{H} 10 \mathrm{~A}$ & 0.9800
\end{tabular}




\begin{tabular}{|c|c|c|c|}
\hline $\mathrm{O} 1-\mathrm{C} 8$ & $1.368(2)$ & $\mathrm{C} 10-\mathrm{H} 10 \mathrm{~B}$ & 0.9800 \\
\hline $\mathrm{O} 1-\mathrm{C} 7$ & $1.382(2)$ & $\mathrm{C} 10-\mathrm{H} 10 \mathrm{C}$ & 0.9800 \\
\hline $\mathrm{C} 1-\mathrm{C} 8$ & $1.361(2)$ & $\mathrm{C} 11-\mathrm{C} 16$ & $1.386(2)$ \\
\hline $\mathrm{C} 1-\mathrm{C} 2$ & $1.448(2)$ & $\mathrm{C} 11-\mathrm{C} 12$ & $1.386(2)$ \\
\hline $\mathrm{C} 2-\mathrm{C} 7$ & $1.392(2)$ & $\mathrm{C} 12-\mathrm{C} 13$ & $1.385(3)$ \\
\hline $\mathrm{C} 2-\mathrm{C} 3$ & $1.395(2)$ & $\mathrm{C} 12-\mathrm{H} 12$ & 0.9500 \\
\hline $\mathrm{C} 3-\mathrm{C} 4$ & $1.382(2)$ & $\mathrm{C} 13-\mathrm{C} 14$ & $1.374(3)$ \\
\hline $\mathrm{C} 3-\mathrm{H} 3$ & 0.9500 & $\mathrm{C} 13-\mathrm{H} 13$ & 0.9500 \\
\hline $\mathrm{C} 4-\mathrm{C} 5$ & $1.394(3)$ & $\mathrm{C} 14-\mathrm{C} 15$ & $1.366(3)$ \\
\hline $\mathrm{C} 5-\mathrm{C} 6$ & $1.387(3)$ & $\mathrm{C} 15-\mathrm{C} 16$ & $1.385(2)$ \\
\hline $\mathrm{C} 5-\mathrm{H} 5$ & 0.9500 & $\mathrm{C} 15-\mathrm{H} 15$ & 0.9500 \\
\hline $\mathrm{C} 6-\mathrm{C} 7$ & $1.385(2)$ & $\mathrm{C} 16-\mathrm{H} 16$ & 0.9500 \\
\hline $\mathrm{O} 3-\mathrm{S} 1-\mathrm{O} 2$ & $119.75(8)$ & $\mathrm{C} 6-\mathrm{C} 9-\mathrm{H} 9 \mathrm{~A}$ & 109.5 \\
\hline $\mathrm{O} 3-\mathrm{S} 1-\mathrm{C} 1$ & $109.59(8)$ & $\mathrm{C} 6-\mathrm{C} 9-\mathrm{H} 9 \mathrm{~B}$ & 109.5 \\
\hline $\mathrm{O} 2-\mathrm{S} 1-\mathrm{C} 1$ & $106.33(7)$ & $\mathrm{H} 9 \mathrm{~A}-\mathrm{C} 9-\mathrm{H} 9 \mathrm{~B}$ & 109.5 \\
\hline $\mathrm{O} 3-\mathrm{S} 1-\mathrm{C} 11$ & $107.27(8)$ & $\mathrm{C} 6-\mathrm{C} 9-\mathrm{H} 9 \mathrm{C}$ & 109.5 \\
\hline $\mathrm{O} 2-\mathrm{S} 1-\mathrm{C} 11$ & $107.36(8)$ & $\mathrm{H} 9 \mathrm{~A}-\mathrm{C} 9-\mathrm{H} 9 \mathrm{C}$ & 109.5 \\
\hline $\mathrm{C} 1-\mathrm{S} 1-\mathrm{C} 11$ & $105.71(8)$ & $\mathrm{H} 9 \mathrm{~B}-\mathrm{C} 9-\mathrm{H} 9 \mathrm{C}$ & 109.5 \\
\hline $\mathrm{C} 8-\mathrm{O} 1-\mathrm{C} 7$ & $107.05(12)$ & $\mathrm{C} 8-\mathrm{C} 10-\mathrm{H} 10 \mathrm{~A}$ & 109.5 \\
\hline $\mathrm{C} 8-\mathrm{C} 1-\mathrm{C} 2$ & $107.60(14)$ & $\mathrm{C} 8-\mathrm{C} 10-\mathrm{H} 10 \mathrm{~B}$ & 109.5 \\
\hline $\mathrm{C} 8-\mathrm{C} 1-\mathrm{S} 1$ & $126.93(13)$ & $\mathrm{H} 10 \mathrm{~A}-\mathrm{C} 10-\mathrm{H} 10 \mathrm{~B}$ & 109.5 \\
\hline $\mathrm{C} 2-\mathrm{C} 1-\mathrm{S} 1$ & $125.40(12)$ & $\mathrm{C} 8-\mathrm{C} 10-\mathrm{H} 10 \mathrm{C}$ & 109.5 \\
\hline $\mathrm{C} 7-\mathrm{C} 2-\mathrm{C} 3$ & $119.54(15)$ & $\mathrm{H} 10 \mathrm{~A}-\mathrm{C} 10-\mathrm{H} 10 \mathrm{C}$ & 109.5 \\
\hline $\mathrm{C} 7-\mathrm{C} 2-\mathrm{C} 1$ & $104.59(14)$ & $\mathrm{H} 10 \mathrm{~B}-\mathrm{C} 10-\mathrm{H} 10 \mathrm{C}$ & 109.5 \\
\hline $\mathrm{C} 3-\mathrm{C} 2-\mathrm{C} 1$ & $135.87(15)$ & $\mathrm{C} 16-\mathrm{C} 11-\mathrm{C} 12$ & $121.04(16)$ \\
\hline $\mathrm{C} 4-\mathrm{C} 3-\mathrm{C} 2$ & $116.27(15)$ & $\mathrm{C} 16-\mathrm{C} 11-\mathrm{S} 1$ & $119.36(13)$ \\
\hline $\mathrm{C} 4-\mathrm{C} 3-\mathrm{H} 3$ & 121.9 & $\mathrm{C} 12-\mathrm{C} 11-\mathrm{S} 1$ & $119.58(13)$ \\
\hline $\mathrm{C} 2-\mathrm{C} 3-\mathrm{H} 3$ & 121.9 & $\mathrm{C} 13-\mathrm{C} 12-\mathrm{C} 11$ & $119.27(17)$ \\
\hline $\mathrm{C} 3-\mathrm{C} 4-\mathrm{C} 5$ & $123.25(16)$ & $\mathrm{C} 13-\mathrm{C} 12-\mathrm{H} 12$ & 120.4 \\
\hline $\mathrm{C} 3-\mathrm{C} 4-\mathrm{Cl1}$ & $118.38(14)$ & $\mathrm{C} 11-\mathrm{C} 12-\mathrm{H} 12$ & 120.4 \\
\hline $\mathrm{C} 5-\mathrm{C} 4-\mathrm{Cl1}$ & $118.37(13)$ & $\mathrm{C} 14-\mathrm{C} 13-\mathrm{C} 12$ & $118.39(18)$ \\
\hline $\mathrm{C} 6-\mathrm{C} 5-\mathrm{C} 4$ & $121.29(16)$ & $\mathrm{C} 14-\mathrm{C} 13-\mathrm{H} 13$ & 120.8 \\
\hline $\mathrm{C} 6-\mathrm{C} 5-\mathrm{H} 5$ & 119.4 & $\mathrm{C} 12-\mathrm{C} 13-\mathrm{H} 13$ & 120.8 \\
\hline $\mathrm{C} 4-\mathrm{C} 5-\mathrm{H} 5$ & 119.4 & $\mathrm{~F} 1-\mathrm{C} 14-\mathrm{C} 15$ & $118.07(18)$ \\
\hline $\mathrm{C} 7-\mathrm{C} 6-\mathrm{C} 5$ & $114.81(15)$ & $\mathrm{F} 1-\mathrm{C} 14-\mathrm{C} 13$ & $118.47(18)$ \\
\hline $\mathrm{C} 7-\mathrm{C} 6-\mathrm{C} 9$ & $122.84(17)$ & $\mathrm{C} 15-\mathrm{C} 14-\mathrm{C} 13$ & $123.45(17)$ \\
\hline $\mathrm{C} 5-\mathrm{C} 6-\mathrm{C} 9$ & $122.34(16)$ & $\mathrm{C} 14-\mathrm{C} 15-\mathrm{C} 16$ & $118.13(18)$ \\
\hline $\mathrm{O} 1-\mathrm{C} 7-\mathrm{C} 6$ & $124.81(15)$ & $\mathrm{C} 14-\mathrm{C} 15-\mathrm{H} 15$ & 120.9 \\
\hline $\mathrm{O} 1-\mathrm{C} 7-\mathrm{C} 2$ & $110.37(14)$ & $\mathrm{C} 16-\mathrm{C} 15-\mathrm{H} 15$ & 120.9 \\
\hline $\mathrm{C} 6-\mathrm{C} 7-\mathrm{C} 2$ & $124.83(16)$ & $\mathrm{C} 15-\mathrm{C} 16-\mathrm{C} 11$ & $119.71(17)$ \\
\hline $\mathrm{C} 1-\mathrm{C} 8-\mathrm{O} 1$ & $110.39(15)$ & $\mathrm{C} 15-\mathrm{C} 16-\mathrm{H} 16$ & 120.1 \\
\hline $\mathrm{C} 1-\mathrm{C} 8-\mathrm{C} 10$ & $133.98(16)$ & $\mathrm{C} 11-\mathrm{C} 16-\mathrm{H} 16$ & 120.1 \\
\hline $\mathrm{O} 1-\mathrm{C} 8-\mathrm{C} 10$ & $115.63(14)$ & & \\
\hline $\mathrm{O} 3-\mathrm{S} 1-\mathrm{C} 1-\mathrm{C} 8$ & $-29.69(17)$ & $\mathrm{C} 1-\mathrm{C} 2-\mathrm{C} 7-\mathrm{O} 1$ & $0.51(17)$ \\
\hline $\mathrm{O} 2-\mathrm{S} 1-\mathrm{C} 1-\mathrm{C} 8$ & $-160.47(15)$ & $\mathrm{C} 3-\mathrm{C} 2-\mathrm{C} 7-\mathrm{C} 6$ & $1.2(2)$ \\
\hline $\mathrm{C} 11-\mathrm{S} 1-\mathrm{C} 1-\mathrm{C} 8$ & $85.62(16)$ & $\mathrm{C} 1-\mathrm{C} 2-\mathrm{C} 7-\mathrm{C} 6$ & $-179.20(15)$ \\
\hline
\end{tabular}




$\begin{array}{llll}\mathrm{O} 3-\mathrm{S} 1-\mathrm{C} 1-\mathrm{C} 2 & 153.51(13) & \mathrm{C} 2-\mathrm{C} 1-\mathrm{C} 8-\mathrm{O} 1 & 0.14(18) \\ \mathrm{O} 2-\mathrm{S} 1-\mathrm{C} 1-\mathrm{C} 2 & 22.72(16) & \mathrm{S} 1-\mathrm{C} 1-\mathrm{C} 8-\mathrm{O} 1 & -177.13(11) \\ \mathrm{C} 11-\mathrm{S} 1-\mathrm{C} 1-\mathrm{C} 2 & -91.19(15) & \mathrm{C} 2-\mathrm{C} 1-\mathrm{C} 8-\mathrm{C} 10 & 179.67(18) \\ \mathrm{C} 8-\mathrm{C} 1-\mathrm{C} 2-\mathrm{C} 7 & -0.40(18) & \mathrm{S} 1-\mathrm{C} 1-\mathrm{C} 8-\mathrm{C} 10 & 2.4(3) \\ \mathrm{S} 1-\mathrm{C} 1-\mathrm{C} 2-\mathrm{C} 7 & 176.93(12) & \mathrm{C} 7-\mathrm{O} 1-\mathrm{C} 8-\mathrm{C} 1 & 0.18(18) \\ \mathrm{C} 8-\mathrm{C} 1-\mathrm{C} 2-\mathrm{C} 3 & 179.10(17) & \mathrm{C} 7-\mathrm{O} 1-\mathrm{C} 8-\mathrm{C} 10 & -179.45(14) \\ \mathrm{S} 1-\mathrm{C} 1-\mathrm{C} 2-\mathrm{C} 3 & -3.6(3) & \mathrm{O} 3-\mathrm{S} 1-\mathrm{C} 11-\mathrm{C} 16 & 13.38(16) \\ \mathrm{C} 7-\mathrm{C} 2-\mathrm{C} 3-\mathrm{C} 4 & -1.0(2) & \mathrm{O} 2-\mathrm{S} 1-\mathrm{C} 11-\mathrm{C} 16 & 143.30(14) \\ \mathrm{C} 1-\mathrm{C} 2-\mathrm{C} 3-\mathrm{C} 4 & 179.57(17) & \mathrm{C} 1-\mathrm{S} 1-\mathrm{C} 11-\mathrm{C} 16 & -103.50(15) \\ \mathrm{C} 2-\mathrm{C} 3-\mathrm{C} 4-\mathrm{C} 5 & 0.2(2) & \mathrm{O} 3-\mathrm{S} 1-\mathrm{C} 11-\mathrm{C} 12 & -164.96(14) \\ \mathrm{C} 2-\mathrm{C} 3-\mathrm{C} 4-\mathrm{C} 11 & -179.55(12) & \mathrm{O} 2-\mathrm{S} 1-\mathrm{C} 11-\mathrm{C} 12 & -35.04(16) \\ \mathrm{C} 3-\mathrm{C} 4-\mathrm{C} 5-\mathrm{C} 6 & 0.6(3) & \mathrm{C} 1-\mathrm{S} 1-\mathrm{C} 11-\mathrm{C} 12 & 78.15(15) \\ \mathrm{C} 11-\mathrm{C} 4-\mathrm{C} 5-\mathrm{C} 6 & -179.72(13) & \mathrm{C} 16-\mathrm{C} 11-\mathrm{C} 12-\mathrm{C} 13 & -0.5(3) \\ \mathrm{C} 4-\mathrm{C} 5-\mathrm{C} 6-\mathrm{C} 7 & -0.4(2) & \mathrm{S} 1-\mathrm{C} 11-\mathrm{C} 12-\mathrm{C} 13 & 177.85(14) \\ \mathrm{C} 4-\mathrm{C} 5-\mathrm{C} 6-\mathrm{C} 9 & -179.76(16) & \mathrm{C} 11-\mathrm{C} 12-\mathrm{C} 13-\mathrm{C} 14 & -0.2(3) \\ \mathrm{C} 8-\mathrm{O} 1-\mathrm{C} 7-\mathrm{C} 6 & 179.28(15) & \mathrm{C} 12-\mathrm{C} 13-\mathrm{C} 14-\mathrm{F} 1 & -179.88(17) \\ \mathrm{C} 8-\mathrm{O} 1-\mathrm{C} 7-\mathrm{C} 2 & -0.44(17) & \mathrm{C} 12-\mathrm{C} 13-\mathrm{C} 14-\mathrm{C} 15 & 0.4(3) \\ \mathrm{C} 5-\mathrm{C} 6-\mathrm{C} 7-\mathrm{O} 1 & 179.86(14) & \mathrm{F} 1-\mathrm{C} 14-\mathrm{C} 15-\mathrm{C} 16 & -179.67(17) \\ \mathrm{C} 9-\mathrm{C} 6-\mathrm{C} 7-\mathrm{O} 1 & -0.8(3) & \mathrm{C} 13-\mathrm{C} 14-\mathrm{C} 15-\mathrm{C} 16 & 0.1(3) \\ \mathrm{C} 5-\mathrm{C} 6-\mathrm{C} 7-\mathrm{C} 2 & -0.5(2) & \mathrm{C} 14-\mathrm{C} 15-\mathrm{C} 16-\mathrm{C} 11 & -0.7(3) \\ \mathrm{C} 9-\mathrm{C} 6-\mathrm{C} 7-\mathrm{C} 2 & 178.89(16) & \mathrm{C} 12-\mathrm{C} 11-\mathrm{C} 16-\mathrm{C} 15 & 0.9(3) \\ \mathrm{C} 3-\mathrm{C} 2-\mathrm{C} 7-\mathrm{O} 1 & -179.08(13) & \mathrm{S} 1-\mathrm{C} 11-\mathrm{C} 16-\mathrm{C} 15 & -177.40(14)\end{array}$

Hydrogen-bond geometry $\left(\AA,{ }^{\circ}\right)$

\begin{tabular}{lllll}
\hline$D-\mathrm{H} \cdots A$ & $D-\mathrm{H}$ & $\mathrm{H} \cdots A$ & $D \cdots A$ & $D-\mathrm{H} \cdots A$ \\
\hline $\mathrm{C} 9-\mathrm{H} 9 C \cdots \mathrm{O} 2^{\mathrm{i}}$ & 0.98 & 2.57 & $3.331(2)$ & 135 \\
$\mathrm{C} 16-\mathrm{H} 16 \cdots \mathrm{O} 3^{\mathrm{ii}}$ & 0.95 & 2.53 & $3.230(2)$ & 130 \\
\hline
\end{tabular}

Symmetry codes: (i) $-x,-y+1,-z+1$; (ii) $-x,-y+2,-z$. 